\title{
TORNIQUETES - MITOS E VERDADES: UMA REVISÃO DE LITERATURA BASEADA EM JOHN KRAGH
}

\author{
Kleber Luiz de Carvalho Dutra ${ }^{1}$
}

\section{RESUMO}

Este trabalho tem como objetivos descrever o detalhamento acerca da instalação do torniquete e propor a implementação de um protocolo de aplicação do dispositivo no atendimento pré-hospitalar. Realizou-se uma revisão de literatura sobre o tema, de janeiro a maio de 2018, nas bases de dados Scielo, Google Acadêmico, Lilacs, CAPES, PubMed, NCBI e NLM, com 36 trabalhos analisados, e possui um caráter propositivo. As evidências científicas resultaram em novas diretrizes que fundamentam a utilização do torniquete. As taxas de sobrevivência foram maiores entre os usos préhospitalar e hospitalar ( $89 \%$ vs $78 \%$ ), com uso antes do choque e após este ( $96 \%$ vs $4 \%$ ) e entre a aplicação do torniquete e sua não utilização ( $87 \%$ vs $0 \%$ ). Os torniquetes usados corretamente, no momento certo e nas vítimas certas, salvaram vidas; então, a questão não deve ser se os torniquetes são ou não bons dispositivos, mas sim como e quando eles devem ser usados. Com esse novo conhecimento, os torniquetes deixaram de ser o item mais controverso no tratamento de acidentes, e tornaram-se uma intervenção préhospitalar importante, evidenciada fortemente para salvar vidas de vítimas com ferimentos graves nos membros.

Palavras-Chave: Atendimento Pré-Hospitalar. Hemorragia. Torniquete;

\footnotetext{
Capitão do Corpo de Bombeiros Militar de Pernambuco, Graduado em Enfermagem pela Faculdade Estácio do Recife. Email: dutra.cbmpe@gmail.com
} 


\title{
TOURNIQUET - MYTHS AND TRUTHS: A LITERATURE REVIEW BASED ON JOHN F. KRAGH
}

\begin{abstract}
This paper aims to describe the details about the tourniquet installation and proposes the implementation of a protocol of this device in prehospital care. A review of the literature, from January to May of 2018, in the databases Scielo, Google Scholar, Lilacs, CAPES, PubMed, NCBI and NLM, with 36 papers analyzed, has a propositional character. The scientific evidence has resulted in new guidelines that support the use of the tourniquet. Survival rates were higher between prehospital and hospital use (89\% vs 78\%), before and after use of tourniquet ( $96 \%$ vs $4 \%$ ) and between the use of the tourniquet and its non-use ( $87 \%$ vs. $0 \%$ ). The tourniquets used correctly, at the right time and in the right victims, saved lives; then the question should not be whether or not tourniquets are good devices, but rather how and when they should be used. With this new knowledge, tourniquets have ceased to be the most controversial item in the treatment of accidents, and have become an important pre-hospital intervention, strongly evidenced to save lives of victims with severe limb injuries.
\end{abstract}

Keywords: Bleeding. Prehospital Care. Tourniquet.

Artigo Recebido em 31/05/2018 e Aceito em 10/09/2018 


\section{INTRODUÇÃO}

As grandes hemorragias decorrentes de lesões severas nos membros continuam a ser a principal fonte de mortes no campo de batalha, e foi observado que o uso do torniquete melhora as taxas de sobrevivência nas vítimas que apresentam traumas importantes nas extremidades (KRAGH et al., 2008). Consoante com o projeto, desenvolvimento, testes e treinamento do Exército Americano em torniquetes para uso no campo de batalha, todo o efetivo militar em ação direta no terreno, quer seja da área de saúde ou não, leva consigo estes dispositivos para contenção de hemorragias (KRAGH et al., 2008).

A hemorragia externa tem sido cada vez mais reconhecida como uma das principais causas de morte potencialmente evitável após uma lesão grave (BULGER et al., 2014). Essa questão foi amplamente abordada pelo Comitê de Atendimento Tático de Acidentes em Combate (TECCC) dos Estados Unidos da América (EUA) em resposta ao aumento da hemorragia externa com risco de vida observada nos conflitos no Iraque e no Afeganistão. A implementação das diretrizes do TECCC para uso do torniquete tem sido associada a uma redução significativa no número de mortes por combate atribuídas à hemorragia nas extremidades. Lições aprendidas com o manejo militar dessas lesões estão começando a ser adotadas na comunidade civil, e as explosões ocorridas na maratona de Boston, EUA, em 2013, destacou esta questão (BULGER et al., 2014).

O torniquete é definido como qualquer dispositivo de constrição, que pode ser improvisado ou fabricado comercialmente, usado como uma tentativa de cessar um intenso sangramento (KRAGH et al., 2008). Ele é um dispositivo colocado ao redor de um membro e pressionado até a eliminação do fluxo arterial. Há equipamentos fabricados comercialmente, e esta deve ser a primeira escolha no momento da utilização, tendo em vista que os torniquetes 
Revista Científica do Corpo de Bombeiros Militar de Pernambuco

Seção 1 - Artigos Técnico Científicos

Artigo publicado no Vol.04 №10 - Edição de JUL a DEZ 2018 - ISSN 2359-4837(online)

Versão on-line disponível em: http://www.revistaflammae.com.

improvisados podem ter sua instalação dificultada e desempenho prejudicado, em virtude do stress da ocorrência e da falta de prática do socorrista (TECCC, 2017). Porém, caso não esteja disponível, deve ser usado o dispositivo improvisado, confeccionado manualmente pelo socorrista (TECCC, 2017).

Kragh e colaboradores (2009) observaram que, historicamente, há alguns mitos acerca das complicações associadas ao uso dos torniquetes, tais como paralisias, necroses e posterior amputação. Estes pesquisadores verificaram que há apenas 02 estudos de coorte retrospectivos sobre o uso do torniquete na emergência, e neles observou-se que os benefícios da sua aplicação superaram em muito os riscos em relação à perda de membros.

Os torniquetes têm pelo menos meio milênio de idade e, no entanto, não foram rotineiramente utilizados pelos militares americanos no início do conflito no Afeganistão em 2001. Em 2014, no entanto, um artigo no Journal of Trauma, que discutia torniquetes, afirmava que eles têm tido sucesso no atendimento ao trauma no Afeganistão e no Iraque, com aplicação direta nos campos de batalha. Com base no trabalho da equipe médica do Exercito Americano, o número de vidas salvas dessa intervenção foi estimado entre 1.000 e 2.000 (HARTFORD CONSENSUS III, 2015).

Os conhecimentos empíricos discutidos no ano de 2001 nos cursos de trauma civil e militar a nível mundial foi de que o uso de um torniquete para controle de hemorragia provavelmente resultaria em amputação do membro lesionado, e que os efeitos prejudiciais dos dispositivos superavam em muito os benefícios. Os resultados dessa mentalidade eram previsíveis (KRAGH et al., 2008). A revisão realizada por Kelly e colaboradores (2008) verificou que as mortes causadas pelos combates nos primeiros anos dos conflitos no Sudoeste da Ásia (Afeganistão e Iraque) constataram que 77 militares americanos haviam sangrado até a morte por ferimentos nas extremidades (membros). Essas mortes representaram $7,8 \%$ de todos os óbitos em combate analisados. Essa incidência de morte por hemorragia nas extremidades ficou 
Revista Científica do Corpo de Bombeiros Militar de Pernambuco

Seção 1 - Artigos Técnico Científicos

Artigo publicado no Vol.04 №10 - Edição de JUL a DEZ 2018 - ISSN 2359-4837(online)

Versão on-line disponível em: http://www.revistaflammae.com.

essencialmente inalterada em relação aos 7,4\% observados no Vietnã, um quarto de século antes (KELLY et al., 2008).

Kalish e colaboradores (2008) descrevem que, no cenário do trauma civil, há um contínuo debate e insegurança sobre o uso do dispositivo, pois a falta de evidências científicas numa situação diferente daquelas de combate em situações de guerra pode desencorajar os socorristas a utilizarem o torniquete no ambiente pré-hospitalar, provavelmente por conta das razões descritas abaixo:

1) A pressão manual normalmente é suficiente para controlar a hemorragia; 2) Os centros urbanos de atendimento ao trauma são normalmente próximos aos locais das ocorrências, o que torna o controle da hemorragia com a compressão direta suficiente para contê-la, até o início do tratamento definitivo; e 3) Torniquetes levam ao aumento das taxas de amputação, complicação isquêmica e disfunção neurológica (KALISH et al., 2008).

Foi realizado um estudo observacional prospectivo no hospital de apoio de combate dos Estados Unidos em Bagdá, no Iraque, de pacientes que fizeram uso de torniquetes no campo de batalha ou no pronto-socorro, com o objetivo de mensurar o seu uso e as complicações que porventura poderiam advir da sua aplicação. A indicação para uso dos torniquetes estava frequentemente associada a lesões em vasos: dos 300 membros em que houve a utilização do recurso de contenção de hemorragia, 87 foram de amputações traumáticas, 86 de lesões vasculares, 81 de fraturas expostas, 45 de lesões de tecidos moles e 01 de lesão por esmagamento (KRAGH et al., 2008).

O Serviço de Emergência Médica Norte-Americano (EMS) publicou um escopo do modelo prático de atendimento às emergências em 2008, e verificou que a aplicação do torniquete deve fazer parte do conjunto de habilidades que um profissional de emergência hospitalar ou pré-hospitalar, que realiza atendimento a vítimas de trauma, deve possuir para colocar em prática durante o seu atendimento. A recente conferência de consenso de Hartford também 
Revista Científica do Corpo de Bombeiros Militar de Pernambuco Seção 1 - Artigos Técnico Científicos

Artigo publicado no Vol.04 №10 - Edição de JUL a DEZ 2018 - ISSN 2359-4837(online)

Versão on-line disponível em: http://www.revistaflammae.com.

estimula o uso civil de torniquetes para o gerenciamento da hemorragia em eventos de tiro ativo. (BULGER, et al., 2014).

Inicialmente, sugere-se que os socorristas pré-hospitalares devam implementar o uso de torniquetes e agentes hemostáticos como parte do tratamento para hemorragia severa. Tais métodos mostraram-se como sendo rápidos e efetivos na cessação e prevenção da hemorragia intensa dos membros, com o uso dos torniquetes, e outros sangramentos externos severos, em que devem ser utilizados os agentes hemostáticos (HARTFORD CONSENSUS III, 2015).

Os maiores achados no estudo são aqueles que o torniquete de emergência usado pelos socorristas foi fortemente associado a vidas salvas, e quanto mais precoce for a sua aplicação, melhor será a sobrevida do paciente, particularmente se este não tiver entrado em choque antes da sua colocação. $\mathrm{O}$ uso de torniquete foi fortemente associado à sobrevida se o choque estivesse ausente no momento do uso (KRAGH, et al., 2009).

A razão pela qual a ausência de choque associada ao uso do torniquete obteve um melhor prognóstico no atendimento pré-hospitalar em relação à emergência hospitalar deve-se ao fato de que o início do choque no APH advém de características fisiológicas, e o tempo de deslocamento ao hospital possui características geográficas. Se a instalação do dispositivo for realizada apenas na emergência hospitalar, em que o trajeto do local da ocorrência até a unidade de saúde dependerá de diversos fatores geográficos (distância, dificuldades de acesso, trânsito), é possível que ocorra o agravamento do estado da vítima, com forte tendência dela entrar em choque (KRAGH et al., 2009).

O uso de torniquetes melhorou a sobrevida do paciente atendido pelo serviço pré-hospitalar, e a sua aplicação nessa situação é necessária para evitar o início do choque hipovolêmico (KRAGH et al., 2009). O uso do dispositivo no pré-hospitalar está associado a melhores taxas de controle da hemorragia, particularmente nos acidentes com maior gravidade de lesões. 
Revista Científica do Corpo de Bombeiros Militar de Pernambuco

Seção 1 - Artigos Técnico Científicos

Artigo publicado no Vol.04 №10 - Edição de JUL a DEZ 2018 - ISSN 2359-4837(online)

Versão on-line disponível em: http://www.revistaflammae.com.

Observou-se que $57 \%$ das mortes poderiam ter sido evitadas pela aplicação precoce do torniquete, e não há relatos significativos de situações adversas relacionadas ao seu uso. Analisou-se, também, um baixo índice de morbidades decorrentes do uso do torniquete, e mesmo aquelas identificadas foram classificadas como de baixa gravidade e temporárias (KRAGH et al., 2012).

A hemorragia é a causa mais comum do choque no paciente de trauma (PHTLS, 2017). O rápido controle da perda sanguínea é um dos objetivos principais no atendimento ao paciente de trauma, pois na etapa "C" do $A B C D E$, devem ser procurados sangramentos externos significativos, e controlá-los $O$ mais rápido possível (PHTLS, 2017). A compressão direta sobre o local do ferimento aumenta a pressão extraluminal e, portanto, reduz a pressão transmural (diferença de pressão dentro do vaso e fora do vaso), o que contribui na diminuição do sangramento (SCHWEITZER et al., 2017).

Os torniquetes usados corretamente, no momento certo e nas vítimas certas, salvaram vidas; então, a questão não deve ser se os torniquetes são ou não bons dispositivos, mas sim como e quando eles devem ser usados. Com esse novo conhecimento, os torniquetes deixaram de ser 0 item mais controverso no tratamento de acidentes; eles se tornaram uma intervenção préhospitalar importante, evidenciada fortemente para salvar vidas de vítimas com ferimentos graves nos membros. Esses desenvolvimentos estimularam a recente reconsideração de um papel seletivo para torniquetes em traumas civis (KRAGH et al., 2012).

A motivação para a realização deste estudo foi a observação da ausência de orientações e peculiaridades acerca da aplicação do torniquete no serviço de Atendimento Pré-Hospitalar (APH), particularmente no Corpo de Bombeiros Militar de Pernambuco. Considerou-se também a dificuldade em se encontrar, na língua portuguesa, estudos que fundamentem e expliquem o passo a passo para uma correta instalação e consequente eficácia dessa técnica de contenção de hemorragias na atividade citada. Sendo assim, buscou-se realizar um levantamento dos estudos, diretrizes e consensos de 
Revista Científica do Corpo de Bombeiros Militar de Pernambuco

Seção 1 - Artigos Técnico Científicos

Artigo publicado no Vol.04 №10 - Edição de JUL a DEZ 2018 - ISSN 2359-4837(online)

Versão on-line disponível em: http://www.revistaflammae.com.

outros países para a realidade do trabalho realizado em Pernambuco e, também, nos demais Estados do Brasil.

\section{OBJETIVOS}

- Realizar uma revisão da literatura sobre o uso do torniquete;

- Descrever os pormenores acerca da sua instalação;

- Propor a implementação de um protocolo de aplicação do torniquete no atendimento pré-hospitalar.

\section{MÉTODOS}

A base metodológica do estudo foi a revisão bibliográfica das principais literaturas relacionadas ao torniquete. Cabe destacar que a maior parte dos estudos encontrados foi apresentada na língua inglesa, em virtude da baixa disponibilidade de materiais na língua portuguesa sobre o tema, especificamente no tocante à explicação da correta técnica de aplicação, bem como das peculiaridades sobre o seu uso.

Foram pesquisados livros, artigos científicos publicados em revistas e anais de congressos e materiais extraídos de reuniões e encontros de comitês de trauma e de cirurgiões, que tratam sobre a viabilidade e aprovação do uso do torniquete no atendimento pré-hospitalar, nas bases de dados Scielo, Google Acadêmico, Lilacs, CAPES, PubMed, NCBI (National Center for Biotechnology Information), e NLM (United States National Library of Medicine), com os descritores "Torniquete", "Atendimento Pré-Hospitalar" e "Hemorragia", e foram encontrados, de acordo com os critérios pré-estabelecidos, 36 estudos que serviram de embasamento para a execução da presente pesquisa.

O estudo também tem características propositivas, tendo em vista que foi realizado um esclarecimento acerca dos passos e parâmetros que devem ser adotados pelos socorristas durante a aplicação do dispositivo de contenção de hemorragias, previsto na literatura, para que haja uma padronização de procedimentos pelos profissionais. 
Revista Científica do Corpo de Bombeiros Militar de Pernambuco

Seção 1 - Artigos Técnico Científicos

Artigo publicado no Vol.04 №10 - Edição de JUL a DEZ 2018 - ISSN 2359-4837(online)

Versão on-line disponível em: http://www.revistaflammae.com.

Para efeitos deste estudo, é importante destacar que, conforme observado na literatura estudada e utilizada como referência, os principais experimentos para a recomendação da utilização do torniquete foram realizados em situações de combate em guerra, em que os militares fazem uso do dispositivo para realizar o controle de uma grande hemorragia. Sendo assim, quando for usado o termo "ambiente militar", deve-se entender que a referência é ao ambiente de campo de guerra, e quando for utilizado o termo "ambiente civil", referimo-nos às situações que não se enquadrem ao anteriormente descrito, mesmo que o socorro seja realizado por militares.

\section{RESULTADOS E DISCUSSÕES}

Nos 36 estudos encontrados, percebeu-se a prevalência de uma produção do autor Dr. John F. Kragh Jr., pesquisador americano, ortopedista e cirurgião, que exerce suas funções na Seção de Controle de Hemorragias, Departamento de Ressuscitação de Controle de Danos - Instituto de Pesquisa Cirúrgica do Exército dos Estados Unidos da América. Por fazer parte desta Instituição, as suas pesquisas sobre torniquete foram fortemente focadas na aplicação do dispositivo em campos de batalha, porém foi verificado que os mesmos princípios utilizados nessa situação podem ser aplicados no que ele chama de "ambiente civil". Observa-se, inclusive, que os seus estudos sobre a aplicação do torniquete são devidamente referenciados pelo PHTLS (Prehospital Trauma Life Support), livro que serve como base para o estabelecimento de doutrinas de Atendimento Pré-Hospitalar.

Nos casos de amputação traumática, em que não há a possibilidade de verificação do pulso mais distal do membro, ou quando existe uma lesão vascular que impossibilite a verificação do pulso distal, utilizou-se apenas o critério de cessação da hemorragia para que o torniquete fosse considerado totalmente efetivo. Deve ser considerada a instalação de um ou mais torniquetes, próximos ao primeiro que foi inicialmente colocado, caso este não tenha apresentado a eficácia desejada (KRAGH et al., 2008). 
Revista Científica do Corpo de Bombeiros Militar de Pernambuco

Seção 1 - Artigos Técnico Científicos

Artigo publicado no Vol.04 №10 - Edição de JUL a DEZ 2018 - ISSN 2359-4837(online)

Versão on-line disponível em: http://www.revistaflammae.com.

Foi analisada também, na literatura, a possibilidade de morbidades associadas ao uso do torniquete, como amputações ou encurtamento dos membros, paralisia, necrose, dores significativas, coágulos, fasciotomia, insuficiência renal aguda, rigor, abscesso, bolhas, abrasões e contusões (KRAGH et al., 2008). A provável explicação para tais lesões pode ser a aplicação incorreta do torniquete, como pressão excessiva, má fixação e afrouxamento não indicado do dispositivo (KRAGH et al., 2008).

Kragh e colaboradores (2008) verificaram que foram aplicados 428 torniquetes em 309 membros lesionados, em 232 pacientes. Aqueles mais efetivos foram os Torniquetes de Emergência Médica - EMT - (92\%) e o Torniquete de Aplicação em Combate - CAT - (79\%), ambos fabricados comercialmente. Quatro pacientes $(1,7 \%)$ sofreram paralisia do nervo transiente no nível do torniquete, enquanto seis tinham paralisia no nível da ferida. Não foi observada relação entre o tempo total do torniquete e morbidade (coágulos, mionecrose, rigor, dor, paralisia, insuficiência renal, amputação e fasciotomia).

Kragh e colaboradores (2008) observaram também que não houve amputações resultantes apenas do uso do torniquete. Entretanto, seis $(2,6 \%)$ vítimas com oito lesões de amputação traumáticas preexistentes tiveram amputações cirúrgicas completas e também tiveram torniquetes aplicados por um período superior a 2 horas. As taxas de membros em que foi necessária a realização de fasciotomia com torniquetes aplicados por menos de 2 horas foi de $28 \%$ ( 75 de 272 ) e mais que 2 horas foi de $36 \%$ (9 de 25 ).

Observou-se que 5 pacientes vieram a óbito por conta da não aplicação dos torniquetes, basicamente porque os socorristas tomaram a decisão de realizar primeiro a extração da vítima do local da ocorrência com posterior transporte (KRAGH et al., 2009), comportamentos esses que vão de encontro à doutrina do $A B C D E$ do trauma, em que o controle da hemorragia com o uso de torniquetes (etapa "C"), precedente às ações anteriormente descritas, poderiam ter salvo a vida dessas cinco vítimas (PHTLS, 2017). 
Revista Científica do Corpo de Bombeiros Militar de Pernambuco

Seção 1 - Artigos Técnico Científicos

Artigo publicado no Vol.04 №10 - Edição de JUL a DEZ 2018 - ISSN 2359-4837(online)

Versão on-line disponível em: http://www.revistaflammae.com.

Diversos tipos de morbidades estiveram associadas ao uso do torniquete, e tais complicações foram devidamente avaliadas. Observou-se a presença de 10 coágulos, estes relacionados a uma trombose venosa profunda e nove trombectomias, realizadas em conjunto com a cirurgia vascular, especialmente quando o reparo do membro era distal. O cirurgião vascular não atribuiu essas trombectomias ao uso do torniquete (KRAGH et al., 2008).

A mortalidade por todas as causas foi de $13 \%$ (31/232) no estudo. Para todas as mortes, a causa primária foi a lesão, e a causa secundária foi a hemorragia, sem mortes atribuídas ao uso do torniquete. Ao analisar o uso do torniquete em relação a uma definição tática de choque (pulso radial fraco ou ausente), 10 pacientes utilizaram torniquete após o seu início, dos quais 9 morreram (90\%) e 222 não apresentavam choque antes do uso do torniquete, dos quais 22 morreram (10\%). Ao analisar o uso do torniquete na emergência hospitalar versus seu uso no pré-hospitalar, 9 de 38 pacientes morreram (24\%) na primeira situação, enquanto que 22 de 194 pacientes pré-hospitalares morreram ( $11 \%$ de mortalidade). A redução pela metade da taxa de mortalidade com uso no pré-hospitalar foi associada também a uma melhora relativa de 16\% na taxa de sobrevida dessas vítimas (KRAGH et al., 2009).

O uso na ausência do choque foi associado com mais vidas salvas do que o uso na presença do choque. $O$ uso no pré-hospitalar foi associado com mais possibilidade de salvar vidas do que a aplicação apenas na emergência hospitalar (KRAGH et al., 2009). Tais achados, provavelmente, podem estar relacionados com a diminuição da taxa de perda de volume sanguíneo e melhora do controle da hemorragia dos membros pelo uso do torniquete no ambiente pré-hospitalar. A agilidade de uma aplicação correta e bem sucedida é vital para a manutenção da vida. O tempo médio de 10 minutos para aplicação do torniquete foi observado, independente da gravidade da lesão, contados do momento da ocorrência até a aplicação do torniquete, e tido como favorável para que não houvesse um risco de morte do paciente. Quando 
Revista Científica do Corpo de Bombeiros Militar de Pernambuco Seção 1 - Artigos Técnico Científicos

Artigo publicado no Vol.04 №10 - Edição de JUL a DEZ 2018 - ISSN 2359-4837(online)

Versão on-line disponível em: http://www.revistaflammae.com.

houver a indicação de uso do torniquete, a sua rápida aplicação deve preceder a extração de uma vítima, bem como o seu transporte (KRAGH et al., 2009).

Observou-se que uma vítima sofreu uma amputação cirúrgica do membro inferior devido a um torniquete deixado no local durante uma longa evacuação para um hospital nacional local com um tempo total de aplicação de 8 horas; após a exploração cirúrgica da perna, não foi encontrada lesão vascular importante. Se o torniquete tivesse sido convertido em curativo hemostático ou sob pressão, durante o Tratamento Tático de Campo (TFC) ou - Tratamento Tático de Evacuação (TACEVAC), seria razoável esperar que a amputação pudesse ter sido evitada (TECCC, 2009).

Foi divulgada, dentro do exército americano, uma diretriz para cuidados prolongados em campos de batalha, e este pode se tornar um referencial importante, porém peculiar, no ambiente civil, pois descreve um procedimento para trocar seguramente torniquetes de membros por curativos locais (hemostáticos) - um processo chamado conversão - que deve ser realizado apenas por pessoal médico qualificado e treinado, nos casos de transporte longo a uma unidade especializada (KRAGH; DUBIK, 2017). Um relato de caso e uma revisão do uso prolongado de torniquetes em um cenário de guerra apontaram a importância da temperatura do membro, porque o aparecimento da isquemia é mais demorado ou evitável se o membro estiver frio (KRAGH; DUBIK, 2017).

Existe a necessidade de um torniquete ser reavaliado no máximo 2 horas após a sua colocação inicial, e serve como um lembrete de que é necessária vigilância para prevenir ou minimizar a morbidade decorrente da aplicação, particularmente quando a evacuação ou o transporte são longos ou tardios (TECCC, 2009). Não houve casos conhecidos de membros perdidos devido à isquemia por conta do torniquete em baixas nos Estados Unidos durante as guerras do Iraque (2003 a 2011) ou Afeganistão (2001 a 2014), embora houvesse pelo menos dois casos não publicados, neste último país, de 
Revista Científica do Corpo de Bombeiros Militar de Pernambuco Seção 1 - Artigos Técnico Científicos

Artigo publicado no Vol.04 №10 - Edição de JUL a DEZ 2018 - ISSN 2359-4837(online)

Versão on-line disponível em: http://www.revistaflammae.com.

perda de membros por torniquetes inadvertidamente deixados no local por longos períodos em vítimas afegãs (TECCC, 2009).

Os curativos compressivos podem ser utilizados, sendo esta a primeira opção para o controle de uma hemorragia. Os torniquetes, por exemplo, controlam $80 \%$ ou mais das hemorragias externas, ocluindo o fluxo arterial, sendo usados amplamente por cirurgiões gerais e ortopédicos estadunidenses por vários anos com resultados satisfatórios. Embora há situações com pequeno risco de uma parte ou todo o membro ser sacrificado, na escolha entre perder um membro ou salvar a vida do paciente, a decisão de preservar a vida deve sempre prevalecer (SCHWEITZER et al., 2017).

A efetividade de controle de uma hemorragia exsanguinante com o uso do torniquete é de $80 \%$ ou mais. Além disso, os torniquetes que impedem o influxo arterial são amplamente usados nas salas de cirurgias há anos, com resultados satisfatórios. Um estudo da atividade militar no Iraque e no Afeganistão demonstrou uma diferença marcante na sobrevida quando o torniquete era aplicado antes do doente entrar em choque descompensado em comparação a quando era aplicado após a queda da pressão arterial (após o choque descompensado). No primeiro caso, a sobrevida foi de $96 \%$; no segundo, de 4\% (PHTLS, 2017).

Relatos antigos de má experiência prática no uso do torniquete delimitaram o uso adequado, porém as evidências científicas resultaram em novas diretrizes que fundamentam a sua utilização, e contribuem com a fabricação comercial de torniquetes, com dispositivos bem projetados e testados. As taxas de sobrevivência foram maiores entre os usos pré-hospitalar e hospitalar ( $89 \%$ vs $78 \%$ ), maiores com uso antes do choque e após este (96\% vs $4 \%$ ) e maiores entre a aplicação do torniquete e sua não utilização (87\% vs $0 \%$ ) (KRAGH et al., 2012). 
Revista Científica do Corpo de Bombeiros Militar de Pernambuco

Seção 1 - Artigos Técnico Científicos

Artigo publicado no Vol.04 №10 - Edição de JUL a DEZ 2018 - ISSN 2359-4837(online)

Versão on-line disponível em: http://www.revistaflammae.com.

\section{CONCLUSÕES E RECOMENDAÇÕES}

O risco de morbidade foi baixo, e houve uma taxa de benefício com relação à sobrevida do paciente. Nenhum membro foi perdido devido ao uso do torniquete, e a duração da sua aplicação não foi associada ao aumento da morbidade. A educação para o uso precoce do torniquete militar deve continuar (KRAGH et al., 2008).

A consciência de que as vítimas podem sangrar rapidamente até a morte em poucos minutos por conta de lesões isoladas nos membros deve ser enfatizada nos treinamentos dos socorristas. Acredita-se que a maioria dos torniquetes que só foram colocados no departamento de emergência deveria ter sido aplicada antes da chegada ao hospital, ainda no atendimento préhospitalar, idealmente antes do início do choque (KRAGH et al., 2009).

Considerando a participação americana na guerra do Afeganistão, e considerando o curto tempo que os militares possuíam para evacuar os locais após uma incursão, os torniquetes foram frequentemente deixados nos membros, no local de aplicação, até o momento em que a vítima estivesse sob os cuidados de um cirurgião. Quando o tempo de evacuação for longo, ocasionando em uma duração também longa de permanência do torniquete, o que é comum nas situações de operações especiais ou de teatros de operações que são ambientes desconhecidos ou de difícil acesso, recomendase converter os torniquetes em curativos hemostáticos, a fim de evitar uma possível isquemia e perda da extremidade. Deve ser destacado que essa técnica de conversão deve ser realizada apenas por equipe médica treinada e especializada (TECCC, 2009).

Estimou-se que 31 vidas foram salvas com o uso do torniquete no préhospitalar, quando comparado com a aplicação apenas no ambiente hospitalar, e o seu uso naquele é claramente justificado e recomendado, pois é evidente que salva mais vidas quando rapidamente aplicado. Atualmente, não há melhor alternativa para se realizar de forma eficaz o estancamento de uma grande 
Revista Científica do Corpo de Bombeiros Militar de Pernambuco

Seção 1 - Artigos Técnico Científicos

Artigo publicado no Vol.04 №10 - Edição de JUL a DEZ 2018 - ISSN 2359-4837(online)

Versão on-line disponível em: $\underline{\text { http://www.revistaflammae.com. }}$

hemorragia em membros do que os torniquetes (KRAGH et al., 2009). Eles são uma medida temporária efetiva, que permite o controle de uma grande hemorragia, e deve ser aplicado, sempre que possível, antes das situações de choque para salvar vidas. Recomenda-se uma política de uso dos torniquetes nas situações de emergência quando há um risco de hemorragia letal dos membros, e sugere-se que seja implementado, nos serviços de atendimento pré-hospitalar, um treinamento sistemático das equipes de socorristas quanto ao uso adequado desta técnica de contenção (KRAGH et al., 2009).

O primeiro passo a ser adotado nos casos de hemorragia severa é a compressão direta no ferimento do membro; caso o sangramento persista após essa ação, então deve ser considerada a aplicação de um torniquete improvisado ou um comercial, e deve ser pressionado até que o sangramento cesse (ATLS, 2018). Um torniquete corretamente aplicado deve ocluir o influxo arterial, pois a oclusão apenas do sistema venoso pode aumentar a hemorragia e resultar em uma extremidade edemaciada e cianótica (ATLS, 2018). O socorrista deve assegurar-se de que o horário de aplicação do torniquete esteja devidamente registrado. Se o tempo de deslocamento do local da ocorrência até uma unidade de saúde for superior a 2 horas, uma única tentativa de conversão ou de afrouxamento do torniquete pode ser considerada em um paciente estável, desde que sob acompanhamento de equipe médica especializada (ATLS, 2018). Os riscos do uso do torniquete aumentam com o tempo; se um torniquete tiver que permanecer no lugar por um período prolongado para salvar uma vida, por conta de fatores diversos, então a manutenção da vida deve prevalecer em relação à preservação da integridade do membro (ATLS, 2018).

Sendo assim, conforme citam Kragh e colaboradores (2008), os seguintes parâmetros e condições devem ser levados em consideração no momento da aplicação de um torniquete: 
Revista Científica do Corpo de Bombeiros Militar de Pernambuco

Seção 1 - Artigos Técnico Científicos

Artigo publicado no Vol.04 №10 - Edição de JUL a DEZ 2018 - ISSN 2359-4837(online)

Versão on-line disponível em: http://www.revistaflammae.com.

1) A aplicação do torniquete antes do aparecimento do choque salva mais vidas do que se instalado após; deve-se usar antes da extração ou do transporte da vítima;

2) Os objetivos do torniquete aplicado no atendimento emergencial são o de estancar a hemorragia e eliminar o fluxo do pulso arterial distal;

3) $O$ uso de um segundo torniquete é útil para a cessação do sangramento e eliminação do pulso distal, quando o primeiro dispositivo instalado não for eficaz no cumprimento desses 02 parâmetros. Ele deve ser colocado mais proximal em relação ao primeiro;

4) Torniquetes têm eficácia quando aplicados proximalmente em relação ao ferimento, mesmo no antebraço ou na perna, e não apenas na coxa ou no braço, como já foi anteriormente recomendado.

Kalish e colaboradores (2008) citam que um ponto crítico na aplicação de qualquer torniquete é que ele seja apertado com pressão suficiente para impedir tanto o fluxo arterial quanto o venoso, e que a parte inferior da perna geralmente não fornece uma boa localização para aplicação de torniquete, pois a tíbia e fíbula impedem a compressão arterial adequada; portanto, um torniquete de coxa distal é tipicamente necessário para lesões poplíteas e mais distais (KALISH et al., 2008).

Esta colocação vai de encontro ao descrito no item "7)" desta lista, que descreve a probabilidade de ineficácia do torniquete no terço distal da coxa, sobre o canal de Hunter, bem como à resposta de Kragh (2018), acerca da aplicação do torniquete na perna (região da tíbia e fíbula):

The evidence I collected was as you say. These segments were the most effective, not the least. This claim of the type stated goes back at least to the 1700's. I cannot find a dataset reported. I take it people saw a case or two or simply repeat the claim without any experience themselves. The operating room tourniquet science squares well with my data-supported findings. That operating room tourniquet science contradicts the claim with which you and I do not agree. I think when the 2-boned segments are injured badly, the wound may disrupt the ligaments at the elbow or wrist which mechanically disrupts the 2boned linkage. In other words, the wound has hidden parts which many people may not detect. They are rarely an orthopedic surgeon like me. So they may have a point, but they may have placed the 
tourniquet atop the wound without realizing it. This is an error in that they attribute the problem to the patient: they blame the victim. This error (misplacement by non-detection of the extent of wounded tissues) may in itself risk ineffectiveness. In my experience and data, this error appears to be an uncommon or rare circumstance if it exists at all. The wound extent experienced by the patient may not square with what an observer sees. If ineffectiveness occurs, knowledgeable users can usually troubleshoot easily. Sometimes, ischemic loss of a joint (by surgical amputation later) like the knee or elbow is risked a little by the practice the claimers suggest (KRAGH, 2018).

As provas que coletei foram como você diz. Esses segmentos (coxa e braço) foram os mais eficazes, porém não os únicos. Essa afirmação, conforme levantamentos, remontam pelo menos aos anos 1700 . Não consigo encontrar um conjunto de dados relatado que justifique tal afirmação. Eu entendo que as pessoas viram um caso ou dois, ou simplesmente repetem a descrição, porém sem nenhuma experiência efetiva. A ciência do torniquete da sala de cirurgia se enquadra bem com os dados por mim coletados. Essa ciência do torniquete da sala de cirurgia contradiz a alegação com a qual você e eu discordamos. Eu acho que quando os segmentos de 2 ossos são feridos gravemente, a ferida pode atrapalhar os ligamentos no cotovelo ou no pulso, o que mecanicamente perturba a articulação de 2 ossos. Em outras palavras, a ferida tem partes ocultas que muitas pessoas podem não detectar. Eles raramente são cirurgiões ortopédicos como eu. Então eles podem ter razão, mas podem ter colocado o torniquete sobre a ferida sem perceber. Este erro (colocação em local inadequado por não detecção da extensão dos tecidos feridos) pode, por si só, causar ineficácia. Na minha experiência e dados, esse erro parece ser uma circunstância incomum ou rara, se existir. A extensão da ferida experimentada pelo paciente pode não se encaixar com o que um observador vê. Se ocorrer ineficácia, os usuários experientes geralmente podem solucionar problemas facilmente. Às vezes, arrisca-se a perda de uma articulação, por isquemia (com posterior amputação cirúrgica), como o joelho ou cotovelo, pela prática sugerida por aqueles que atestam a ineficácia de um torniquete aplicado no antebraço ou na perna, regiões dos membros com 2 ossos (TRADUÇÃO LIVRE DO AUTOR).

5) Os torniquetes devem ser aplicados a cerca de 5 a $7 \mathrm{~cm}$ acima do ferimento;

6) Se for utilizado um torniquete improvisado, a bandagem deve ser dobrada para que fique com uma largura de no mínimo 5 e no máximo $10 \mathrm{~cm}$;

7) Deve ser evitada a aplicação do torniquete sobre o canal de Hunter, localizado no terço inferior da coxa, próximo ao joelho, por haver risco de ineficácia do dispositivo, tendo em vista que há um canal entre o músculo e o 
Revista Científica do Corpo de Bombeiros Militar de Pernambuco

Seção 1 - Artigos Técnico Científicos

Artigo publicado no Vol.04 №10 - Edição de JUL a DEZ 2018 - ISSN 2359-4837(online)

Versão on-line disponível em: http://www.revistaflammae.com.

osso, o que poderá ocasionar uma impossibilidade de compressão dos grandes vasos.

O Canal de Hunter é um canal formado pela aponeurose de inserção do adutor magno na diáfise femoral. Quando essa aponeurose se insere, deixa um canal entre o músculo e o osso. Tem como limites: Anterior - costureiro (sartório), Posterior- Adutor Magno, e Lateral - vasto-medial. Pode ser definido também como um canal musculoaponevrótico que constitui a porção inferior do canal femoral e que se estende ao longo de todo o terço inferior da coxa. Por ele passam a artéria femoral, a veia femoral e o nervo safeno interno (FORTIN; UHL; GILLOT, 2013).

8) As roupas devem ser removidas sempre que possível, para possibilitar a visualização de todas as lesões;

9) Adereços devem ser retirados, bem como qualquer tipo de material sob o torniquete, a fim de evitar sua frouxidão;

10) O torniquete pode ser aplicado sobre as roupas, caso não seja possível retirá-las, porém isso pode dificultar a visualização do ponto de hemorragia. Neste caso, o dispositivo deve ser colocado o mais proximal possível do membro afetado (técnica "alto e apertado"). Se for possível retirar as vestimentas, com consequente visualização do ferimento, então o torniquete deve ser colocado a cerca de 5 a $7 \mathrm{~cm}$ acima da lesão;

11) $O$ torniquete não deve ser instalado nas articulações, sob risco de ineficácia;

12) É preferível o uso de torniquetes comerciais, pois são projetados cientificamente, testados em laboratório e validados clinicamente;

13) Use torniquetes improvisados, instalados manualmente, quando os fabricados comercialmente não estiverem disponíveis;

14) $O$ monitoramento do torniquete aplicado deve ser uma adoção primordial, tendo em vista que, com uso prolongado, haverá a cessação do fluxo sanguíneo para a área abaixo da lesão, o que poderá resultar na diminuição da 
Revista Científica do Corpo de Bombeiros Militar de Pernambuco

Seção 1 - Artigos Técnico Científicos

Artigo publicado no Vol.04 №10 - Edição de JUL a DEZ 2018 - ISSN 2359-4837(online)

Versão on-line disponível em: http://www.revistaflammae.com.

circunferência do membro, com consequente afrouxamento do dispositivo e possível retorno da hemorragia;

15) A educação, o treinamento e a doutrina do torniquete são ações vitais e devem ser divulgadas e refinadas, sempre baseadas em evidências;

Para efeitos de recomendação da presente pesquisa, quando se fala em aplicação do torniquete na região abaixo do joelho, percebe-se que não há um consenso entre os autores, e nem estudos que fundamentem ou não, cientificamente, a sua aplicação nessa região. Sendo assim, até que novos experimentos sejam realizados e divulgados, sugere-se inicialmente a execução das ações previstas por John Kragh, pesquisador que embasa este trabalho (aplicação do torniquete na região da tíbia e da fíbula), e caso não surta o efeito desejado, então se aplica um segundo torniquete na região medial da coxa.

Quando a situação e o tempo permitirem, a verificação do pulso distal deve ser realizada nos membros em que houver um torniquete aplicado. Se o pulso distal ainda estiver presente, então a pressão do torniquete deve ser aumentada, ou, caso haja o risco de lesão, um segundo torniquete deve ser aplicado, lado a lado e acima do primeiro, a fim de eliminar o pulso distal (TECCC, 2017).

Sugere-se, então, o passo a passo abaixo na instalação de um torniquete para a contenção de hemorragias no atendimento pré-hospitalar: 


\section{Revista FLAMMAE}

Revista Científica do Corpo de Bombeiros Militar de Pernambuco

Seção 1 - Artigos Técnico Científicos

Artigo publicado no Vol.04 №10 - Edição de JUL a DEZ 2018 - ISSN 2359-4837(online)

Versão on-line disponível em: http://www.revistaflammae.com.

\section{Quadro 1 - Passo a passo para a instalação de um torniquete} improvisado.

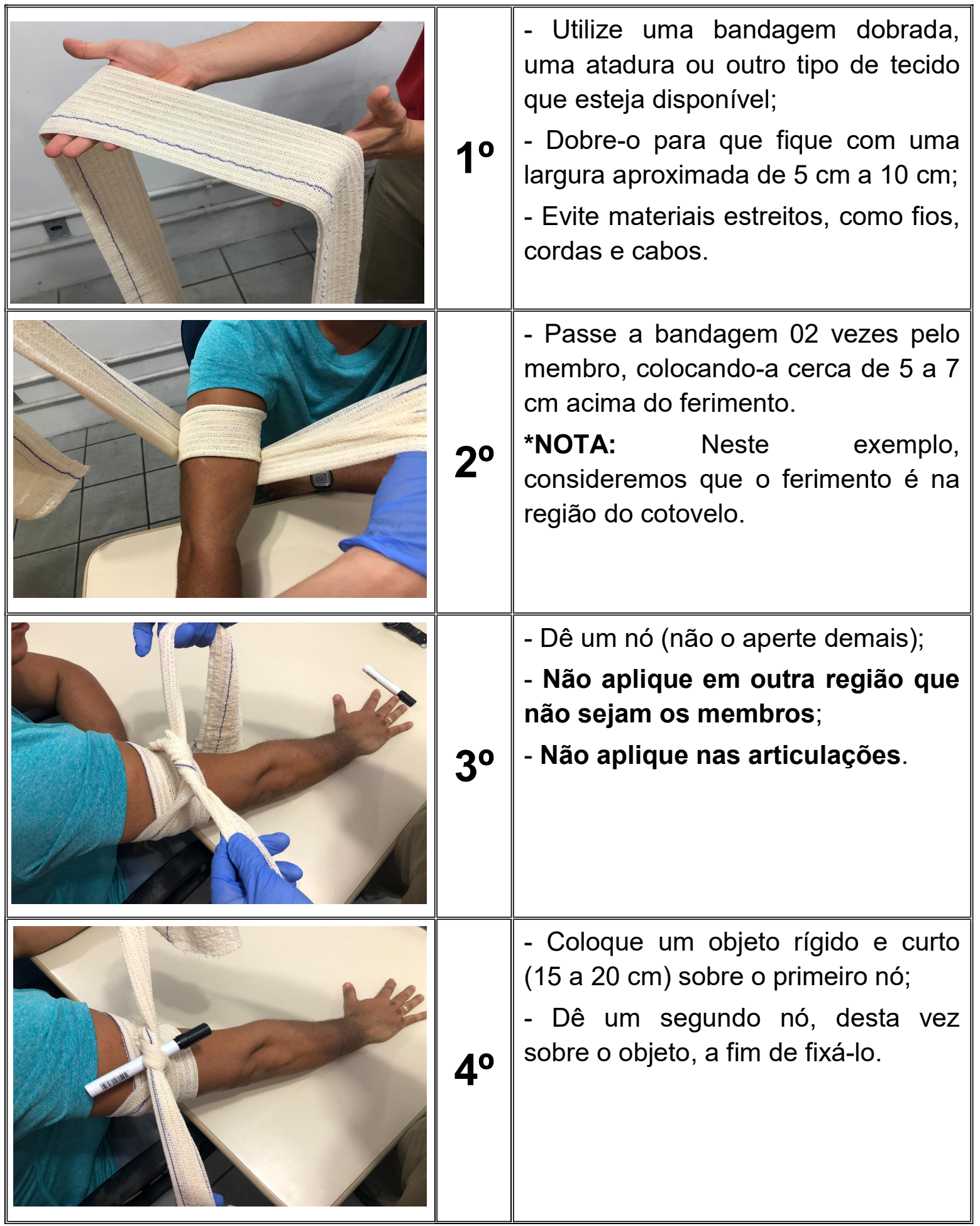




\section{Revista FLAMMAE}

Revista Científica do Corpo de Bombeiros Militar de Pernambuco

Seção 1 - Artigos Técnico Científicos

Artigo publicado no Vol.04 №10 - Edição de JUL a DEZ 2018 - ISSN 2359-4837(online)

Versão on-line disponível em: http://www.revistaflammae.com.

\begin{tabular}{|c|c|c|}
\hline & $5^{0}$ & $\begin{array}{l}\text { - Gire o objeto (no sentido horário ou } \\
\text { anti-horário) até que a hemorragia } \\
\text { cesse e até a eliminação do pulso } \\
\text { distal; } \\
\text { - Cuidado: Caso um desses } \\
\text { parâmetros não seja atingido com } 01 \\
\text { torniquete, o socorrista não deve } \\
\text { forçá-lo, sob risco de lesão vascular } \\
\text { e tecidual. } \\
\text { - Fixe o objeto com a própria atadura } \\
\text { (ou bandagem), para que ele não } \\
\text { afrouxe. }\end{array}$ \\
\hline & & $\begin{array}{l}\text { *NOTA: Caso um dos parâmetros } \\
\text { descritos no item anterior não seja } \\
\text { atingido (cessação da hemorragia } \\
\text { e/ou eliminação do pulso distal), } \\
\text { então um segundo torniquete deve } \\
\text { ser aplicado, desta vez acima do } \\
\text { primeiro. } \\
\text { Neste exemplo, o segundo } \\
\text { dispositivo seria aplicado na região } \\
\text { entre o ombro e o primeiro } \\
\text { torniquete. }\end{array}$ \\
\hline & $6^{\circ}$ & $\begin{array}{l}\text { - Escreva a sigla "TQ" (para } \\
\text { torniquete), juntamente com o } \\
\text { horário de aplicação, em um } \\
\text { esparadrapo (ou fita), e coloque na } \\
\text { bandagem ou atadura; } \\
\text { - Não afrouxe o torniquete; } \\
\text { - Não cubra nem o torniquete e } \\
\text { nem o ferimento. }\end{array}$ \\
\hline
\end{tabular}

Fonte: THYGERSON et al., 2017.

Imagens: Do autor. 
Revista Científica do Corpo de Bombeiros Militar de Pernambuco

Seção 1 - Artigos Técnico Científicos

Artigo publicado no Vol.04 №10 - Edição de JUL a DEZ 2018 - ISSN 2359-4837(online)

Versão on-line disponível em: http://www.revistaflammae.com.

Acredita-se que torniquetes devidamente aplicados podem, com segurança, rapidez e eficácia, controlar adequadamente o risco de morte em virtude de uma hemorragia decorrente de uma lesão penetrante em uma extremidade, e esta intervenção deve ser mais rotineiramente considerada, especialmente em sistemas pré-hospitalares, nos quais uma intervenção imediata contribui sobremaneira para a manutenção da vida e consequente sobrevida do paciente (KALISH et al., 2008).

Kragh e colaboradores (2008) descrevem que os torniquetes podem ser ferramentas poderosas para o bem e para o mal. Bons porque salvam vidas quando usados na hora certa, da maneira certa, porém se forem usados na hora errada e de maneira errada, podem trazer complicações para a vítima. $O$ uso de torniquetes após a extração, transporte ou choque, ou distalmente em relação à ferida, causou aumento da mortalidade nos pacientes. Os benefícios superaram em muito os riscos durantes os experimentos, sem perda de membros apenas por conta do uso do torniquete. Muitas vidas foram salvas pelo uso de torniquetes pré-hospitalares em comparação à aplicação apenas em ambiente hospitalar. Os dispositivos são uma medida temporária que permite o controle eficaz da hemorragia, e devem ser aplicados precocemente, antes que a vítima esteja em estado de choque para salvar vidas. Um torniquete não reverte o choque, mas pode atenuá-lo, com ganho de tempo vital para que o socorrista possa iniciar uma reanimação com maiores chances de sucesso (KRAGH et al., 2008).

\section{AGRADECIMENTOS}

Ao Sr. Maj QOC/BM Cristiano Corrêa, exemplo a ser seguido, pelo constante estímulo à produção científica; 
Revista Científica do Corpo de Bombeiros Militar de Pernambuco

Seção 1 - Artigos Técnico Científicos

Artigo publicado no Vol.04 №10 - Edição de JUL a DEZ 2018 - ISSN 2359-4837(online)

Versão on-line disponível em: http://www.revistaflammae.com.

Aos amigos e companheiros de turma, Capitães QOC/BM Quintino e Rafael, e ao amigo Sgt BM Vandson, pela contribuição nas fotografias apresentadas neste estudo.

\section{REFERÊNCIAS}

AMERICAN COLLEGE OF SURGEONS. Advanced Trauma Life Support. Chicago: 2018.

AMERICAN COLLEGE OF SURGEONS. The Hartford Compendium Strategies to Enhance Survival in Active Shooter and Intentional Mass Casualty Events: A Compendium. Chicago: 2015.

BULGER, E. M. et al. An evidence-based prehospital guideline for external hemorrhage control: American College of Surgeons Committee on Trauma. Prehospital Emergency Care, v. 18, n. 2, p. 163-73, abr./jun. 2014.

FORTIN, C.; UHL, J.; GILLOT, C. Le canal de Hunter: un dispositif de régulation du retour veineux póplite? Intérêts pour les phlébologue. La Revue Internationale de Phlébologie, v. 66, n. 3, p. 39-45, 2013.

$\mathrm{KALISH}$, J. et al. The return of tourniquets. Original research evaluates the effectiveness of prehospital tourniquets for civilian penetrating extremity injuries. The Journal of Emergency Medical Services, v. 33, n. 8, p. 44-54, ago. 2008.

KELLY, J. F. et al. Injury severity and causes of death from Operation Iraqi Freedom and Operation Enduring Freedom: 2003-2004 vs 2006. The Journal of Trauma, v. 64, n. 2, p. 21-26, fev. 2008 (edição suplementar).

$\mathrm{KRAGH}$, J. F. et al. Battle casualty survival with emergency tourniquet use to stop limb bleeding. The Journal of Trauma Medicine, v. 41, n. 6, p. 590-97, dez. 2009.

KRAGH, J. F.; DUBICK, M. A. Bleeding control with limb tourniquet use in the wilderness setting: review of science. Wilderness and Environmental Medicine, v. 28, n. 2, p. 25-32, jun. 2017 (edição suplementar).

$\mathrm{KRAGH}$, J. F. et al. Historical review of emergency tourniquet use to stop bleeding. The American Journal of Surgery, v. 203, n. 2, p. 242-52, fev. 2012.

$\mathrm{KRAGH}, \mathrm{J}$. F. et al. Practical use of emergency tourniquets to stop bleeding in 
major limb trauma. The Journal of Trauma, Injury, Infection and Critical Care, v. 64, n. 2, p. 38-50, fev. 2008 (edição suplementar).

$\mathrm{KRAGH}, \mathrm{J}$. F. et al. Survival with emergency tourniquet use to stop bleeding in major limb trauma. Annals of Surgery, v. 249, n. 1, p. 1-7, jan. 2009.

$\mathrm{KRAGH}$, J. F. et al. Torniquetes [mensagem pessoal]. Mensagem recebida por <dutra.cbmpe@gmail.com> em 30 jun. 2018.

NATIONAL ASSOCIATION EMERGENCY MEDICAL TECHNICIANS. Prehospital Trauma Life Support (PHTLS). $8^{\mathrm{a}}$ ed. Burlington: Jones \& Bartlett, 2017.

SCHWEITZER, G. et al. Intervenções de emergência realizadas nas vitimas de trauma de um serviço aeromédico. Revista Brasileira de Enfermagem, $v$ 70, n. 1, p. 48-54, jan./fev 2017.

TACTICAL EMERGENCY CASUALTY CARE COMITTEE (TECCC). Guidelines for BLS/ALS Medical Providers. Tampa: 2017.

TACTICAL EMERGENCY CASUALTY CARE COMITTEE (TECCC). Optimizing the use of Limb Tourniquets in Tactical Combat Casualty Care. Texas: 2009.

THYGERSON, A. L. et al. First Aid, CPR and AED - Advanced. $7^{\mathrm{a}}$ ed. Jones \& Bartlett, 2017. 